\title{
Efficient Robust Watermarking of Compressed 2-D Grayscale Patterns for H.264/AVC
}

\author{
Jing Zhang and Anthony T. S. Ho \\ Center for Information Security \\ School of Electrical and Electronic Engineering \\ Nanyang Technological University \\ Singapore 639798 \\ Email: Jingzhang@pmail.ntu.edu.sg; etsho@ntu.edu.sg
}

\begin{abstract}
An efficient and robust video watermarking algorithm for the state-of-the-art video coding standard H.264/AVC is proposed for copyright protection. Grayscale 2-D watermark patterns such as detailed trademarks or logos can be highly compressed by a proposed grayscale watermark pre-processing, and inserted into the low bit-rate H.264/AVC videos in the compressed domain. The marked video sequences maintain good visual quality and the same overall consuming bit-rate. The proposed algorithm can robustly survive transcoding process and common signal processing, such as bit-rate reduction, Gaussian filtering and contrast enhancement.
\end{abstract}

Index Terms-H.264/AVC, robust video watermarking, grayscale watermark pre-processing, compressed 2-D grayscale patterns, copyright protection.

\section{INTRODUCTION}

The progress in multimedia storage and transmission technology has allowed an ever increasing amount of information in digital format for archiving and transmission. However, the ease of copying and reproducing digital data is more likely to encourage the work of pirates and hackers, since it enables perfect copies with no loss in data quality. Digital watermarking represents a valid solution to the above problem, since it is possible to identify the source, author, creator, owner, distributor or authorized consumer of digitized images, video recordings or audio recordings. A digital watermark is an identification code, permanently embedded into digital data, carrying information pertaining to copyright protection.

Digital video watermarking algorithms can be sorted according to the embedding domain, such as the spatial domain, compressed domain, and bitstream domain. Compresseddomain watermarking methods arouse great interests among researchers. Hartung et al. [1] embedded spread-spectrum watermark into the MPEG-2 bitstream with drift compensation, but the capacity is reduced. Arena et al. [2] watermarked the MPEG-2 video using interleaved coding. The differential energy watermark method has been extended to video from still images by watermarking the I-frames of an MPEG stream as in [3]. However, most of the previous works were focusing on MPEG standards for high bit-rate applications rather than low bit-rate videos $(\leq 1 \mathrm{Mbit} / \mathrm{s})$.

The H.264/AVC standard is specially designed for low bitrate applications. It significantly improves the coding efficiency with the help of many enhanced functional features, such as the highly flexible tree-structured motion compensation, superior rate-distortion related Lagrangian Optimization, $4 \times 4$ Integer DCT and the very efficient contextbased arithmetic-coding scheme [4]. H.264/AVC has achieved averagely $50 \%$ in bit-rate savings when compared with existing standards such as MPEG-4 Video [4]. However, higher compression ratio leads to the difficulty in balancing among tradeoff requirements for watermarking video data [5], [6].

This paper describes a digital watermarking scheme of H.264/AVC video format, working directly in the compressed domain. The scheme makes an accurate usage of the video encoder for watermark embedding, and experimental results exhibit robust results for this low bit-rate format under transcoding and common signal processing. In the digital rights management system, company trademarks and logos are always considered as excellent watermark for copyright protection [5]. Hence, this paper also proposes a novel preprocessing for grayscale watermark patterns. It is more important that the pre-processing can help to solve the low capacity problem for watermarking the H.264/AVC video due to the high compression performance.

The grayscale watermark pre-processing method is introduced in the next section. The proposed watermarking method based on the emerging H.264/AVC standard will also be described. The simulation results and conclusions will be given in Section III and Section IV, respectively.

\section{Proposed Watermarking Algorithm}

In this paper, 2-D texture grayscale trademarks and logos are first pre-processed and then embedded as the robust watermarks for copyright protection for H.264/AVC video data. Figure 1 shows the watermarking embedding scheme consisting of grayscale watermark pre-processing and H.264/AVC encoder with watermark embedding.

\section{A. Grayscale Watermark Pre-processing}

The advancement in video coding technology has led to a significant decrease in redundancy for watermark embedding. For example, in bi-predicted frames of a video clip with low motion at a low coding bit-rate, most coefficients after prediction are zero [6]. Accordingly, the watermark should be pre-processed to decrease the data size for embedding. A 

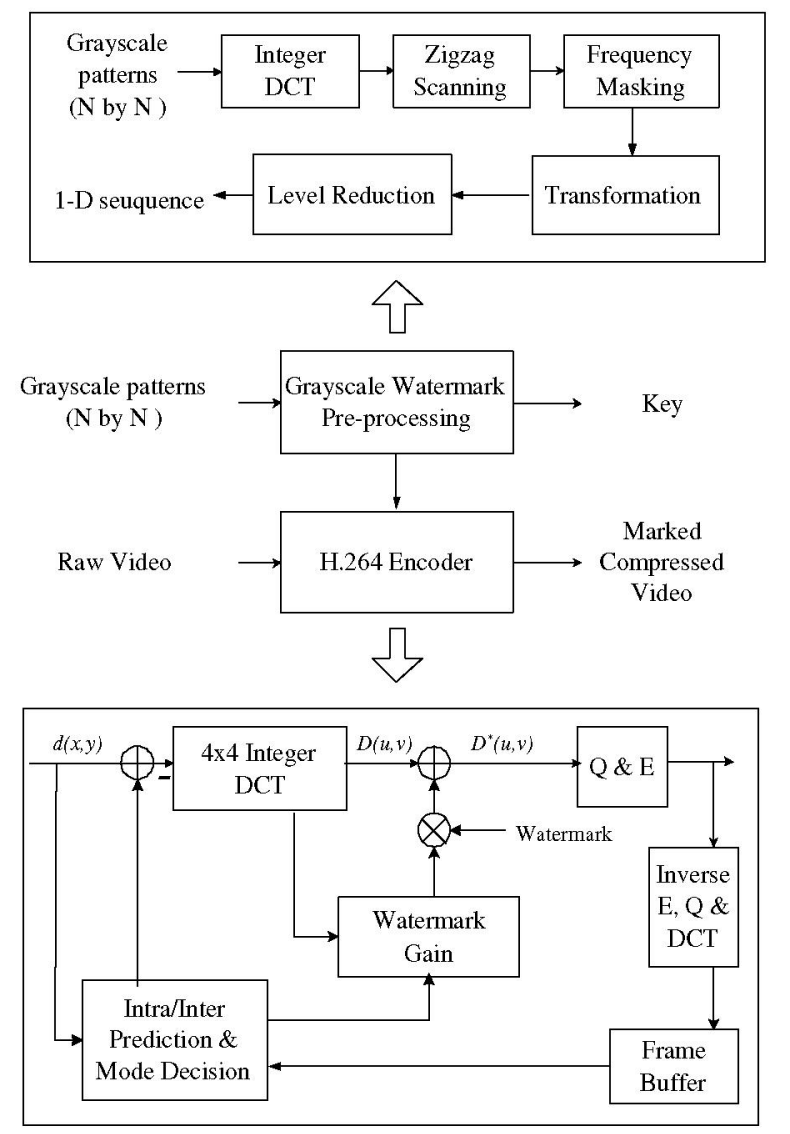

Fig. 1. Watermark Embedding Scheme with H.264/AVC Encoder.

grayscale watermark pre-processing is proposed to deal with this problem.

1) $4 \times 4$ Integer DCT: The grayscale pattern is first decomposed into non-overlapping $4 \times 4$ blocks, denoted by $m(x, y)$ $(0 \leq x, y \leq 3)$. Each block $m(x, y)$ is transformed by

$$
M(u, v)=\operatorname{DCT}\{m(x, y)\} \quad 0 \leq u, v \leq 3
$$

where DCT $\{\cdot\}$ represents the less complex but more accurate integer DCT with the fixed block-size $4 \times 4$ which is uniquely used in H.264/AVC [4].

2) Zigzag Scanning and Frequency Masking: After zigzag scanning, from the $4 \times 4$ transformed block we obtain 16 coefficients $M(j)$, where $j \in[0,15]$ indicates the position in zigzag scanning. There are several lowest frequencies which possess the significant energy with sufficient information for the pattern reconstruction. To achieve higher efficiency, a mask is applied to select the top 6 coefficients and discard the other components.

3) Transformation: After masking, there is a significant difference between the dynamic range of every two adjacent coefficients. Thus, the remaining coefficients $M(j)$ are transformed to reduce the dynamic range as follows:

$$
M^{\prime}(j)=\frac{M(j)+A(j)}{B(j)} \quad 0 \leq j<15
$$

where $A(j)$ and $B(j)$ represent the corresponding transformation parameters to $j$, where $j \in[0,15]$ indicates the position in zigzag scanning.

4) Level Reduction: The coefficients $M^{\prime}(j)$ after transformation have multiple values. They can be further simplified to be binary, denoted by $w(j)$ as

$$
\begin{gathered}
w(j) \equiv\left[M^{\prime}(j)\right]_{\bmod 2} \\
K(j)=M^{\prime}(j) / 2
\end{gathered}
$$

Watermark patterns can then be reconstructed from the 1-D output sequence $w$ obtained from the pre-processing and the key file $K$.

After the grayscale watermark pre-processing, a 2-D grayscale watermark pattern with the size $N \times N$ (with $N \times N$ spacial pixels) can be compressed into a binary sequence $w$ with the shortest length $3 N^{2} / 8$. This significantly decreases the watermarking payload for every frame by approximately $63 \%$.

\section{B. H.264/AVC Watermarking Method}

1) Watermark Embedding: In the next stage, the binary sequence $w$ is first mapped into a bipolar sequence and then modulated by the Spread-Spectrum method in [7] to obtain the watermark sequence for embedding, denoted by $w^{s}$.

Due to the significant compression efficiency of the H.264/AVC standard, the remaining DCT coefficients of the residual data are perceptually essential. Thus, during the H.264/AVC encoding process, only one middle frequency $D(u, v)$ in the diagonal position in a $4 \times 4$ DCT block is substituted by

$$
D^{*}(u, v)=\alpha \cdot \beta_{i} \cdot w_{i}^{s}
$$

where $u$ and $v$ indicate the position of the coefficient, $w_{i}^{s}$ is the spread-spectrum watermark component, both $\alpha$ and $\beta_{i}$ are positive gain factors to maintain a good visual quality of the marked video, and $i$ denotes the position of the block, $i \in R$.

The embedded watermark $w_{i}^{s}$ is a bipolar component. Thus, from Equ. 5, the watermark information is embedded into the polarity the frequency component by replacing operation, instead of modulating the amplitude in traditional addition embedding methods:

$$
\operatorname{sign}\left(D^{*}(u, v)\right)=w_{i}^{s}
$$

During video processing and transcoding process, the amplitude of the H.264/AVC compressed video data can be altered in a very wide dynamic range. Therefore, embedding watermark components into the data polarity can help increase the robustness of the watermarking scheme.

The gain factor $\alpha$ is derived empirically. The local gain factor $\beta_{i}$ is obtained from the DC coefficient $D(0,0)$ and the $\mathrm{AC}$ coefficients $D(u, v)$ :

$$
\beta_{i}=\mu \sum_{\substack{1 \leq u \leq 3 \\ 1 \leq v \leq 3}}|D(u, v)|-D(0,0)
$$


where $\mu$ is the the weighting factor which balances the DC and $\mathrm{AC}$ coefficients.

From this formula, the local watermark gain is determined by the local motion activity from the integer DCT coefficients of the residual error. The DC coefficient represents the luminance of the block, and the sum of the $\mathrm{AC}$ coefficient $D(u, v)$ represents the spatial activity of the block. The local gain factor can alter adaptively according to the local motion activity. The local watermark gain can be linked with perceptual masking adaptively with the visual content.

To increase compression efficiency, the H.264/AVC standard supports a tree-structured motion compensation [4] with 7 Inter-prediction modes for each macroblock $S$ as well as two Intra-prediction modes: $16 \times 16,16 \times 8,8 \times 16,8 \times 8,8 \times 4$, $4 \times 8$ and $4 \times 4$ for Inter-prediction, $I 16 \times 16$ and $I 4 \times 4$ for Intra-prediction. A larger partition size is suitable for still areas and a smaller partition size is suitable for areas with detailed motion.

After embedding $w_{i}^{s}$ into all the available Inter/Intra modes (denoted by $\mathcal{I}$ ), the best mode $o^{*}$ for the marked macroblock $M B^{*}$ is selected by minimizing the expression in Eq. (8) within the constrained $R$ and minimized $D$, using Lagrangian Optimization technique [4] of H.264/AVC:

$$
o^{*}=\arg \min _{o \in \mathcal{I}}\left(D\left(M B^{*}, o\right)+\lambda R\left(M B^{*}, o\right)\right)
$$

where $\lambda$ denotes the predetermined Lagrangian multiplier for mode choice, and $D$ and $R$ represent the distortion and consumed bits for encoding the current mode $o$, respectively.

2) Watermark Retrieval: For extraction, the original embedded watermark components are not needed. At the H.264/AVC Decoder, one watermark coefficient $\hat{w}_{i}^{s}$ can be estimated by the sign of the corresponding marked quantized coefficient $\hat{D}_{q}(u, v)$ in each $4 \times 4$ block:

$$
\hat{w}_{i}^{s}=\operatorname{sign}\left(\hat{D}_{q}(u, v)\right) \quad 0 \leq u, v \leq 3, i \in R
$$

The Hamming distance $H$ between the extracted watermark $\hat{w}^{s}$ and the original spread-spectrum chip sequence is computed. The extracted bit $\hat{w}_{i}$ can be derived from the Hamming distance with an appropriate threshold $\tau . \hat{w}_{i}=1$ if $H>\tau$; $\hat{w}_{i}=0$ if $H<-\tau$.

\section{Grayscale Watermark Post-precessing}

After extracting the sequence $\hat{w}_{i}$ from the compressed H.264 video, the grayscale watermark is reconstructed by performing an inverse operation of the watermark pre-processing.

\section{Results And Analysis}

In our experiments, the proposed H.264/AVC watermarking algorithm has been integrated into the H.264 JM-7.6 reference software [8]. The standard test video clips include Foreman, Stefan, Coastguard, Flower Garden, Container Ship, and Silent. All the first 100 frames are coded at 15 frames/s at $768 \mathrm{kbits} / \mathrm{s}, 512 \mathrm{kbits} / \mathrm{s}$ and $396 \mathrm{kbist} / \mathrm{s}$, respectively.
OK
OK $(\rho=0.9482)$
OK $(\rho=0.9327)$
(a) Original
(b) Reconstructed
(c) Extracted

Fig. 2. Samples of The Original, Reconstructed and Extracted Watermarks from the 30th Frame of Stefan (768 kbit/s, CIF-size) and Normalized Correlation.

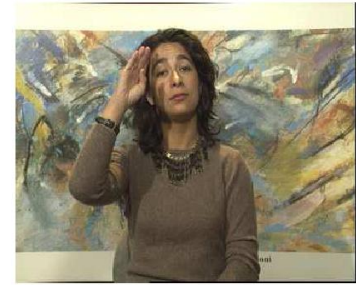

(a) Unmarked (41.82dB)

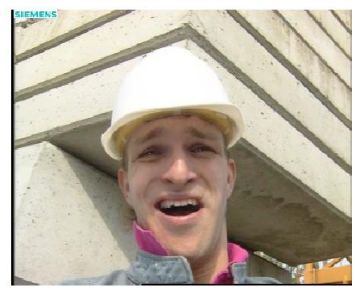

(c) Unmarked ( $41.55 \mathrm{~dB})$

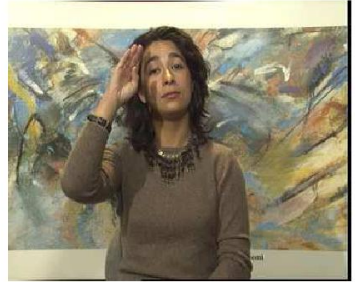

(b) Marked (39.52dB)

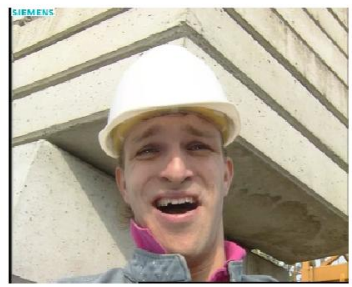

(d) Marked (41.00dB)
Fig. 3. The 20th frames of unmarked and markedSilent((a) and (b)) and Foreman((c) and (d)) $(768 \mathrm{kbit} / \mathrm{s}, \mathrm{CIF}-\mathrm{size})$.

A small $16 \times 16$ grayscale watermark is used as the watermark pattern for our experiments. Figure 2 shows the reconstructed watermark without watermarking and the retrieved samples from the marked Stefan ( $768 \mathrm{kbit} / \mathrm{s}, \mathrm{CIF}$-size). These reconstructed watermark samples are highly correlated to the original pattern. This demonstrates and supports the feasibility of the proposed grayscale watermark pre-processing technique.

Figure 3 shows the frame samples from the unmarked and marked video clips with PSNR(dB). The unmarked and marked video clips are reconstructed from the compressed data without and with watermarks, respectively. The average PSNR values are computed by comparing the reconstructed video frames to the original raw video frames.

On average, the watermark insertion leads to a decrease of approximately $0.97 \mathrm{~dB}$ for all video clips coded at $768 \mathrm{kbits} / \mathrm{s}$, $0.96 \mathrm{~dB}$ at $512 \mathrm{kbits} / \mathrm{s}$ and $0.64 \mathrm{~dB}$ at kbits $/ \mathrm{s}$, which are all less than $1 \mathrm{~dB}$. In the experiments, no visible artifacts can be observed in all of the test video sequences.

Two categories of video attacks have been applied to the marked video to test the robustness of the watermarking algorithm: transcoding and common signal processing processes.

In the first group, we utilized the bit-rate reduction. During watermarking in the H.264/AVC encoding process, the Lagrangian Optimization technique targets the overall consuming bit-rate at $768 \mathrm{kbits} / \mathrm{s}, 512 \mathrm{kbits} / \mathrm{s}$ and $396 \mathrm{kbits} / \mathrm{s}$. When transcoding is applied after watermarking, the bit-rates have 
been reduced to approximately the $1 / 2$ and even the $1 / 3$ of the original bit-rates. During re-encoding with the bit-rate reduction, most high frequency components which represent detailed texture will be discarded.

After decoding the marked bitstream, common signal processing attacks are applied to the raw video frame by frame, including $5 \times 5$ Gaussian low-pass filtering, circular averaging filtering, unsharpened contrast enhancement, and additive Gaussian noise $($ mean $=0$, variance $=0.001$ ). Then the attacked raw video is coded again. Thus, there are actually two attacks applied each time: decoding and re-encoding, and common signal processing.

The robustness is measured by the normalized correlation between the original grayscale watermark and the extracted and results are presented in Figure 4.

Figure 4 shows that the proposed watermarking algorithm together with the watermark pre-processing can survive strong attacks, such as bit-rate reduction, contrast enhancement and Gaussian noise. The watermark patterns can tolerate the distortion introduced by some incorrect extracted watermark components, and perform better than binary watermark sequences. Moreover, the fact that the watermark components are embedding into the polarities of the DCT coefficients of the residual data, instead of modifying the amplitude also play an important role in increasing the robustness of the algorithm. Further more, by applying the spread-spectrum to the pre-processed watermark components before embedding repeatedly spreads the watermark components into every macroblock of each frame. This also increases the robustness of our algorithm.

\section{Conclusions}

The proposed grayscale watermark pre-processing with the presented video watermarking algorithm for copyright protection technique performed well in terms of robustness and data capacity. The watermarked H.264/AVC video clips maintained the good visual quality and almost the same Bit-rate. Other more detailed copyright information, such as textured company trademarks or logos can also be used as watermark to further protect ownership and defend against illegal attacks.

\section{REFERENCES}

[1] F. Hartung and B. Girod, "Watermarking of uncompressed and compressed video," Signal Processing, vol. 66, no. 3, pp. 283-301, May 1998.

[2] S. Arena, M. Caramma and R. Lancini, "Digital watermarking applied to MPEG-2 coded video sequences exploiting space and frequency masking," The IEEE International Conf. on Image Processing, pp. 1987 1990, Sep. 2000.

[3] G. Langelaar and R. Lagendijk, "Optimal differential energy watermarking of DCT encoded images and video," IEEE Trans. Signal Processing: Image Commun., vol. 10, pp. 148-158, 2001.

[4] T. Wiegang, G.J. Sullivan, G. Bjøntegaard, and A. Luthra, "Overview of the H.264/AVC video coding standard," IEEE Trans. Circuits Syst. Video Technol., vol. 13, no. 7, pp. $560-576$, Jul. 2003.

[5] J. Zhang and A.T.S. Ho, "An efficient digital image-in-image watermarking algorithm using the integer discrete cosine transform (IntDCT)," in IEEE Joint Conf. of 4th Int. Conf. Info., Commun. and Signal Processing and 4th Pacific-Rim Conf. Multimedia, Dec. 2003.

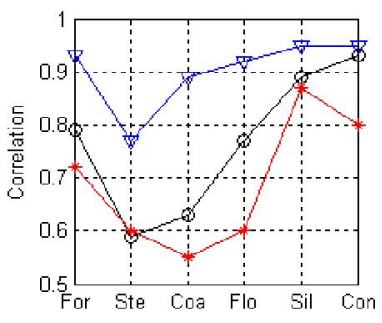

(a) No attacks

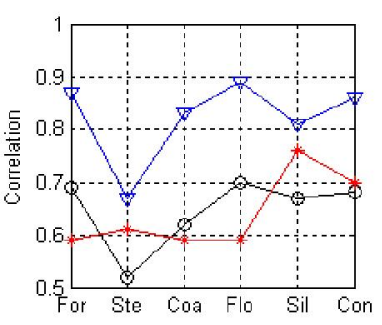

(c) $5 \times 5$ Gaussian Filtering

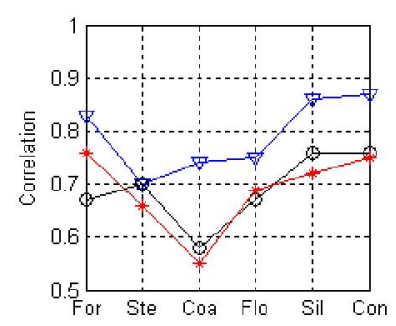

(e) Contrast Enhancement

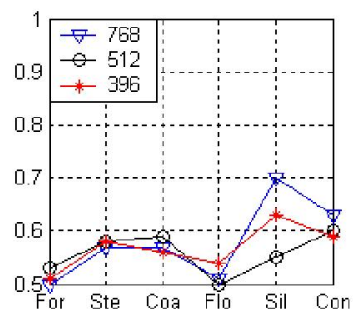

(b) To $1 / 3$ Bit-rate

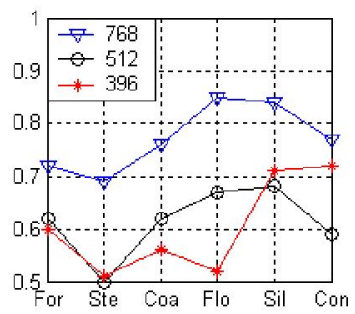

(d) Circular Filtering

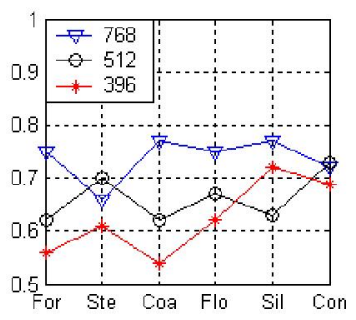

(f) Gaussian Noise
Fig. 4. Robustness under transcoding and common signal processing on Foreman, Stefan, Coastguard, Flower, silent and Container (denoted by For, Ste, Coa, Flo, Sil and Con in the horizontal axis) (768 kbit/s, $512 \mathrm{kbit} / \mathrm{s}$, and $396 \mathrm{kbit} / \mathrm{s}, \mathrm{CIF}-\mathrm{size})$.

[6] G. Qiu, P. Marziliano, A.T.S. Ho, D.J. He, and Q.B. Sun, "A hybrid watermarking scheme for H.264/AVC video," in Proc. the 17th International Conference on Pattern Recognition, Cambridge, UK, Aug. 2004.

[7] A.T.S. Ho, and F. Shu, "A Robust Spread-Spectrum Watermarking Method Using Two-Level Quantization," in IEEE International Conference on Image Processing (ICIP 2004), Singapore, Oct. 2004.

[8] H.264/AVC Joint Model 7.6 (JM-7.6) Reference Software. [Online]. Available: http://iphome.hhi.de/suehring/tml/download/old_jm/. 